American J. of Engineering and Applied Sciences 1 (4): 389-392, 2008

ISSN 1941-7020

(C) 2008 Science Publications

\title{
Intelligent Estimation of Compressive Strength of the Pavement Layers Stabilized by the Combination of Bitumen Emulsion and Cement
}

\author{
Mehrdad Aryafar, Abdoul R. Ghotbi, Mehdi Aryafar and Amin Avaei \\ Department of Civil Engineering, Shahid Bahonar University, \\ 22 Bahman Blvd, Kerman, Kerman, Iran
}

\begin{abstract}
The Application of the different types of additive materials such as lime, cement bitumen and the combination of them are considered as a main issue by the relating experts. In order to promote the bearing capacity of road, these materials, individually, or with the attendance of other materials add to sub base layers. During the recent years, road builders have been considering the application of the combination of bitumen emulsion and cement due to the emergence of the modern equipments and machineries in transportation engineering which have been led to the rapid construction of roads and a uniform combination with the suitable compactness properties in soil stabilization too. The compressive strength which can be determined by the Unconfined Compressive Strength (UCS) test is one of the most important factors to control the quality of the stabilized materials using bitumen emulsion and cement and also in order to design them much efficiently. Besides, it is necessary to use an analytical method because the laboratory tests are very expensive and in some cases are not available especially in the projects constructing in the remote areas and also the strong need for controlling the obtained results from the insitu tests. In this study, the application of the inelegant neural network is investigated to estimate the 28 days compressive strength of the samples built from the stabilized materials by the combination of bitumen emulsion and cement. The obtained results show that; artificial neural network is very capable in predicting the 28 days compressive strength.
\end{abstract}

Key words: Artificial neural network, compressive strength, network teaching, unconfined compressive strength (UCS)

\section{INTRODUCTION}

The knowledge of artificial network has had a considerable progress during the recent 15 years. The basic researches were begun in the mid 19 century by Ivan Pavlov and consequently were peruse by the scientists such as, Warren Mc Culloch and Walter Pitts in 1943, Donald Hebb in 1949, Frank Rosenblatt in 1958 and Bernurd Widrow, Marvin Minsky, Seymour Papert in the mid decade of 60B.C and finally by John Hopfield, David Rummelhart and James Mcland by 1982 to 1985 up to now ${ }^{[1,2]}$.

Using the interpretation of the empirical data, artificial neural network conducts the knowledge or the rule hidden behind the data to network structure and despite the mathematical models, there is no need to determine a mathematical relation between the input and the output values. So that in cases which it is too difficult to imply a complicated relation between the variables especially in the physical terms, artificial neural network is very capable. On the other hand, in the mathematical functions, the incorrect input values or the incomplete ones cause a very significant error in the output values while neural network presents a better output results close to the exact results ${ }^{[3]}$.

Considering that the laboratory tests are so expensive especially in the field of transportation projects and due to the shortage of the required models for estimating the strength properties of the materials stabilized by the combination of bitumen emulsion and cement, it is so necessary to do further researches to achieve the mentioned goals. In order to access this propose, using 97 tests done on the constructed samples with different percentages of cement and bitumen emulsion, the compressive strength is stimulated and is modeled by artificial neural network. The presented model can be effective for estimating the compressive strength and can decrease the costs of the laboratory tests.

\section{MATERIALS AND METHODS}

Artificial neural network: The main body of each artificial neural network includes some joins and the

Corresponding Author: Ghotbi, A., Faculty of Civil Engineering, Shahid Bahonar University, 22 Bahman Blvd, Kerman, Iran Tel/Fax: +98(21)-33939450 
relevant connections. The joins are the neural compiler which is called neuron. The network connections have their own weight which show the amount of the cooperation between two neurons and would be variable. The network weight, indeed, shows the network memory, so it cab be said that the network science is distributed in the whole network. Therefore, they can be called the distributed associative memory. The transfer functions can be used to conduct the output values from each layer to another one ${ }^{[1,4]}$. In this research, the feed forward multilayer perceptron network has been used. The feed forward multineural network has at least an input layer and an output layer or several hidden layers. In these networks, the neurons have been placed in the parallel layers and there is no connection between the joins of a layer. The neurons of each layer just connect to the neurons of the layers located in two sides of them and this connection is a unilateral one. The neurons in a layer just transmit the signal to the neurons in the next layer. These networks have an input layer or an output layer or several hidden layers at least. The performance of the networks is estimated using Mean Square Error (MSE). The process in which the weights and the bias are changed until MSE to be minimized is called the network teaching rule. In order to teach the network. In the present research, the back propagation algorithm is used as well as the numerical optimization Levenberg-Marquardt technique ${ }^{[1]}$.

Useful data for network teaching: The base materials are produced by the combination of bitumen emulsion and cement ${ }^{[5]}$. The compressive strength of the material depends on the percentage of the applied cement and the bitumen emulsion. The input values include the percentage of bitumen emulsion and cement. So that the number of the neurons of the input layers is 2 and considering that the output values of the network are the compressive strength of the stabilized base materials, the number of the relevant neurons is just 1 . In order to teach the network, 97 unconfined compressive tests have been done which the results of 64 tests are used for teaching the network and others are used for the simulation.

\section{Network teaching:}

Determination of the best rule for teaching: The network teaching is the process in which the weights and the bias of the network change until minimize the network error. The back propagation algorithm has been used for the network teaching. This rule is similar to the teaching rule of mean square error of the steepest decent algorithm (SD). The difference between the back propagation algorithm and the Least Mean Square (LMS) is related to the type of differentiation.

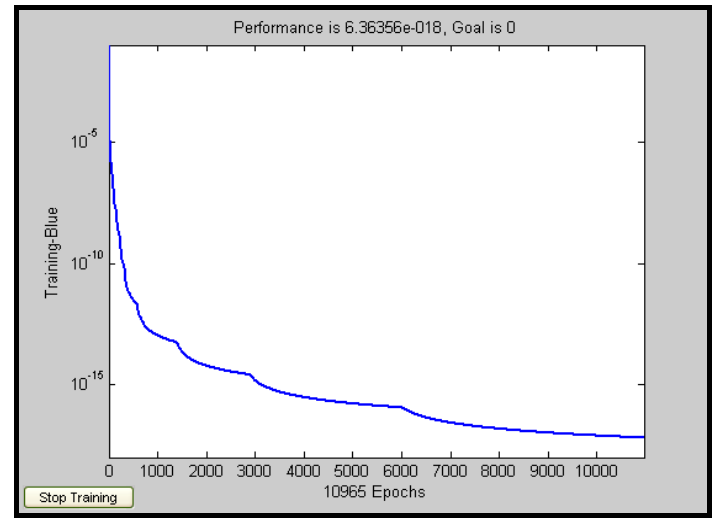

Fig. 1: The process of network teaching using Momentum method

In the linear networks including one layer, it is used from LMS. In these networks, error is the obvious function of the network weights and its differentiation can be calculated easily with respect to the weights, but in the multilayer networks including the nonlinear transfer functions, there are the complicated relations between the weights and the network errors and it is required to use the chain rule for calculating the differentiations. So that the back propagation algorithm, in fact, is the application of the minimum square method to the multilayer networks with the nonlinear functions. The Sigmooied tangant transfer function (TANSIGN) has been used for the presently applied network for the first layer (hidden layer) and the Sigmooied logarithm transfer function has been used for the second layer ${ }^{[1]}$. As it can be shown in Fig. 1, in the Momentum method, the teaching function remains constant after a reduction Also and finally in cycle of 20000, the network teaching is stopped. Using the simulation results, it can be concluded that; although the teaching error is 0.000567045 , the network has not been converged yet and the teaching process has not been successful. In the next step, in order to teach the network, it is used from the numerical optimization system of Levenberg-Marquardt. Figure 2, shows the process of the network teaching using this technique. As it is so obvious in Fig. 2, in the cycle of 20000, the error of the network teaching is about $3.6682 \mathrm{E}-16$ and the simulation results indicate the excellent network teaching.

Determination of the best structure: After determination of the best teaching rule, the number of the neurons in the hidden layer is optimized for reaching to the minimum error ${ }^{[6]}$. The teaching process and the network error are investigated using the large number of the neurons. The number of the hidden layers is shown respectively in Fig. 3-5. 
Am. J. Engg. \& Applied Sci., 1 (4): 389-392, 2008

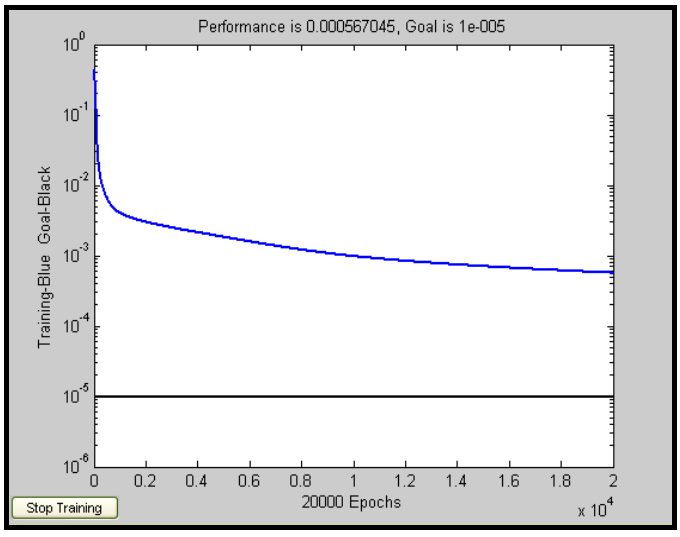

Fig. 2: The process of network teaching using the Levenberg and Marquardt technique

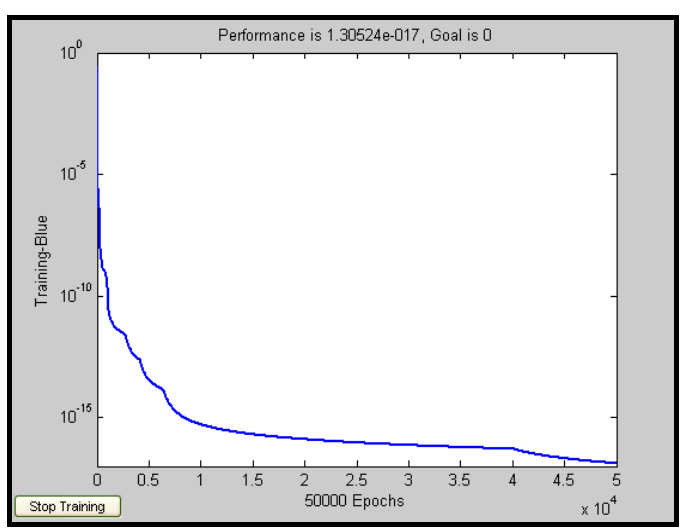

Fig. 3: The teaching process using 19 neurons in the hidden layer

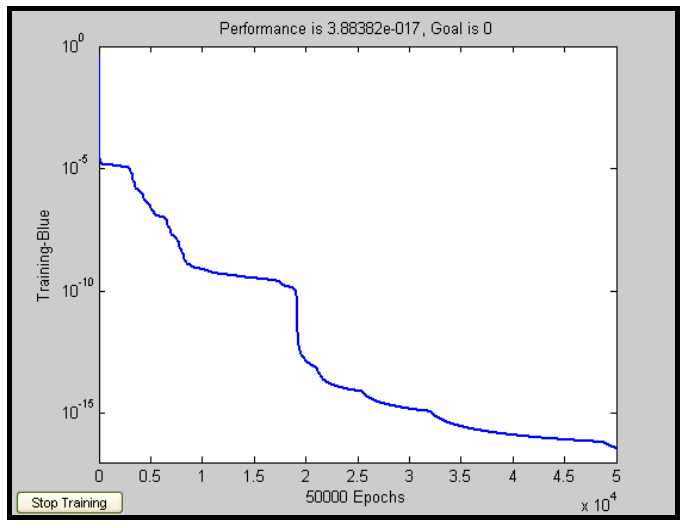

Fig. 4: The teaching process using 26 neurons in the hidden layer

The mentioned figures are shown the process of the network teaching using 24, 33 and 28 neurons in the hidden layers.

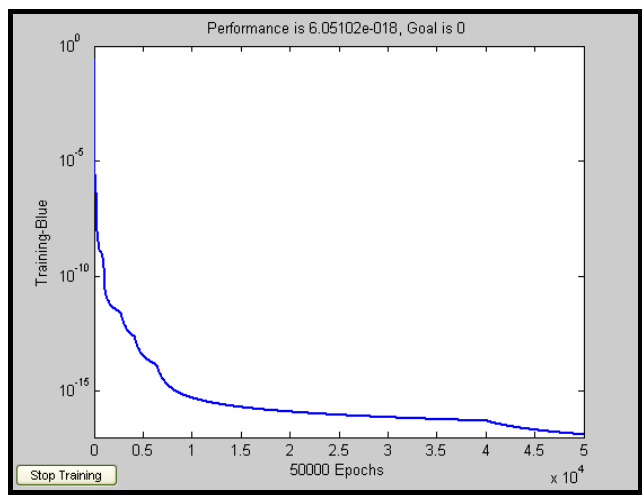

Fig. 5: The teaching process using 28 neurons in the hidden layer

Table 1: The obtained results by the network teaching using various neurons in the hidden layer

\begin{tabular}{llll}
\multicolumn{4}{c}{ neurons in the hidden layer } \\
\hline $\mathrm{N}$ & 19 & 26 & 28 \\
\hline MSE & $1.31 \mathrm{E}-17$ & $3.88 \mathrm{E}-17$ & $6.05 \mathrm{E}-18$ \\
EPOCHS & 50000 & 50000 & 50000 \\
\hline
\end{tabular}

The teaching results are shown in Table $1 . \mathrm{N}$ shows the number of the neurons in the hidden layer. The results show that, using the Levenberg and Marquardt algorithm, the network teaching can easily be done by 19 to 26 neurons in the hidden layer and using this algorithm leads to a good capability of the network teaching using the numerous neurons in the hidden layer and this is one of the most important advantages of this technique. Of course, using 28 neurons, the reduction of the error process gets much quick and as a result, the teaching time reduces. So, for this propose, it has been used from 28 neurons in the hidden layer.

\section{RESULTS AND DISCUSSION}

Comparison of the results obtained by the network teaching with those obtained by the realy stabilzed comperessive strength of the base materials: In order to have much obvious assessment in the results obtained by the network teaching, 18 output values (out of 97) have been given to the network including the experimental results which are shown in Table 2. As it can easily be shown in Table 2, the network simulation using the input values which the network has previously been taught with them, has been done much properly and the errors are not substantial.

The Network teaching and comparison of the obtained results using the simulation of the both network and experimental data: After the end of the network teaching, the relevant test has been done using 33 remained data. The results of the comparison are shown in Fig. 6. The plotted curve in Fig. 6 shows that, despite the existence of some disturbances among the data, there is a really suitable model among the simulated results and the experimental results as it can be mentioned as fallows: 
Am. J. Engg. \& Applied Sci., 1 (4): 389-392, 2008

Table 2: Comparison of the results obtained by the network teaching with the real amounts using the Levenberg and Marquardt technique

\begin{tabular}{|c|c|c|c|}
\hline Sample No. & Output & Real output & Error \\
\hline 1 & 0.2218200 & 0.188110 & 0.0933840 \\
\hline 2 & 0.2519700 & 0.231620 & 0.0503280 \\
\hline 3 & 0.3920400 & 0.389400 & 0.0047020 \\
\hline 4 & 0.6563400 & 0.601720 & 0.0705280 \\
\hline 5 & 0.9984900 & 0.900430 & 0.0913710 \\
\hline 6 & 0.0008118 & 0.012408 & 0.0626140 \\
\hline 7 & 0.0445860 & 0.049632 & 0.0226890 \\
\hline 8 & 0.0762600 & 0.086857 & 0.0408130 \\
\hline 9 & 0.2271900 & 0.231000 & 0.0094419 \\
\hline 10 & 0.4001700 & 0.402880 & 0.0047152 \\
\hline 11 & 0.5127800 & 0.523280 & 0.0150960 \\
\hline 12 & 0.5715100 & 0.574750 & 0.0043460 \\
\hline 13 & 0.0797110 & 0.087316 & 0.0292400 \\
\hline 14 & 0.1105600 & 0.117650 & 0.0243980 \\
\hline 15 & 0.1460400 & 0.147980 & 0.0060293 \\
\hline 16 & 0.2458000 & 0.253680 & 0.0184720 \\
\hline 17 & 0.3997900 & 0.406860 & 0.0122030 \\
\hline 18 & 0.5893800 & 0.579660 & 0.0129240 \\
\hline
\end{tabular}

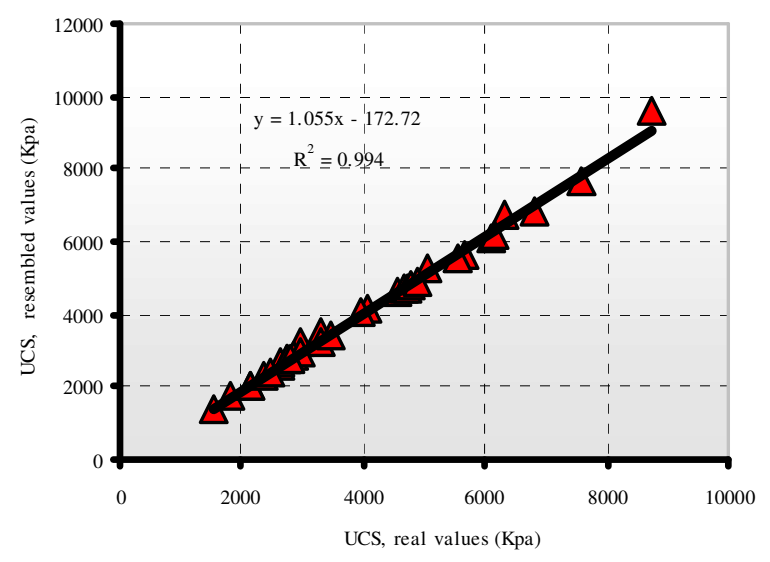

Fig. 6: The comparison of the results obtained by the simulation and the experimental method (kpa)

$$
\mathrm{y}=1.055 \mathrm{x}-172.72
$$

The presented linear model shows that; the simulated results are about 99 percent of the experimental results and the regression coefficient $\left(\mathrm{R}^{2}\right)$ is about 0.994 . It is obvious that using further data for teaching the network promotes its ability.

\section{CONCLUSION}

- Determination of the compressive strength of the base materials by artificial neural network using feed forward multilayer perceptron and with the help of the back propagation algorithm with the numerical Levenberg and Marquardt optimization system could be successful using the lower error of teaching
- In the neural teaching done by the back propagation method, using the numerically modifying Levenberg and Marquardt technique provides a good capability of the network optimization using the numerous neurons of the hidden layer

- The small amount of the error obtained by the MSE method for network teaching dose not verify a good network teaching and simulation capability, as using the Momentum method has not been successful; however using the simulation technique of Levenberg and Marquardt, the network gets much capability for simulating the results

- With increasing the experimental data for teaching the network, the network abilities promote for simulating the results

- The successful simulation of the unconfined compressive strength of the stabilized base materials using artificial neural network is very promising especially for applying the intelligent systems as the supplementary and the controller and even as an alternative for the experimental systems in transportation projects which it mainly leads to reduction of the high costs and also promotes the controlling capability for the projects constructing in the remote area and saves time and money

\section{REFERENCES}

1. Hagan, M.T. and H.B. Demuth, 2000. Neural Network Design. Mark Beale MHB, Inc., PWS Publishing Company.

2. Bart Kosko, Lotfi A. Zade and James A. Anderson, 1992. Neural Networks and Fuzzy Systems. Prentice-Hall Inc.

3. Neil, N., E. Ahmed and B. Senouc, 1995. Condition rating of rigid pavements by neural networks. Can. J. Civil Eng., 22: 861-870.

4. Abdelrahim, A.M. and K.P. George, 2000. Artificial neural network for enhancing selection of pavement maintenance strategy. Transport. Res. Record, 1699: 16-22.

5. Yoder, W., 1975. Principles of Pavement Design. 2nd Edn., John Wiley and Sons, Inc.

6. Owusu-Ababio, S., 1998. Effect of neural network topology on flexible pavement cracking prediction. Comput. Aided Civil Infrastruct. Eng., 13: 349-355. 